\title{
Diagnostic value of clinical deep tendon reflexes in diabetic peripheral neuropathy
}

Zhen-Fei Li ${ }^{1,2}$, Xiao-Li Niü, Ling-Ling Nie4, Li-Ping Chen ${ }^{1,2}$, Cui-Fang Cao ${ }^{1,2}$, Li Guo ${ }^{1,2}$

'Department of Neurology, The Second Hospital of Hebei Medical University, Shijiazhuang, Hebei, China

${ }^{2}$ Key Laboratory of Hebei Neurology, Shijiazhuang, Hebei, China

${ }^{3}$ Department of Neurology, Hebei General Hospital, Hebei, China

${ }^{4}$ Hebei Maternity Hospital, Hebei, China

Submitted: 20 May 2020

Accepted: 27 September 2020

Arch Med Sci

DOI: https://doi.org/10.5114/aoms.2020.100656

Copyright @ 2020 Termedia \& Banach

\section{Abstract}

Introduction: The aim of the present study was to evaluate the diagnostic efficacy of different tendon reflexes in detecting diabetic peripheral neuropathy (DPN).

Material and methods: According to the changes in tendon reflexes, all patients with diabetes were divided into three strata: impaired Achilles reflex only, impaired lower extremity reflexes, and impaired lower and upper extremity reflexes. Taking nerve conduction studies (NCS) as the gold standard, the sensitivity, specificity, and predictive ability of the tendon reflexes of these three strata, as well as the Toronto clinical scoring system (TCSS) and Michigan Neuropathy Screening Instrument (MNSI), were calculated. Then, the electrophysiological characteristics of diabetic patients with different tendon reflexes were analysed.

Results: Among the 240 patients studied, 92 (38.3\%) presented evidence of neuropathy, which was confirmed by abnormal NCS, while 148 (61.7\%) had normal NCS results. Taking NCS as the gold standard, stratum 1 yielded a sensitivity and specificity of $93.5 \%$ and $54.7 \%$, respectively, while stratum 3 had higher specificity (96.6\%) and lower sensitivity (34.8\%) when compared to stratum 1. However, stratum 2 had the highest specificity $(75.7 \%)$. Conclusions: The assessment of tendon reflexes can be proposed as a test for screening diabetic polyneuropathy.

Key words: peripheral neuropathy, deep tendon reflexes, nerve conduction studies, diabetes mellitus.

\section{Introduction}

Diabetic peripheral neuropathy (DPN) is one of the most common complications of diabetes mellitus, and its prevalence has been estimated to be within the range $13-68 \%$ in patients with longstanding diseases [1]. DPN is closely correlated to neuropathic foot ulceration [2]. Furthermore, DPN is the leading cause of non-traumatic limb amputation [3]. Hence, if DPN can be diagnosed and treated early, the incidence of foot ulceration can be reduced.

Dyck $[4,5]$ proposed that abnormalities in two of five criteria ( $\geq 2$ abnormalities from the neuropathy symptom score (NSS), neuropathy disability score (NDS), nerve conduction (NC), quantitative sensory examina-

\author{
Corresponding author: \\ Li Ping Chen \\ Department of Neurology \\ The Second Hospital \\ Hebei Medical University \\ 215 Hepingxi St \\ Xinhua District \\ Shijiazhuang \\ Hebei 050000, China \\ Phone: +86100311 \\ 66003942 \\ Fax: +86 10031166003942 \\ E-mail: \\ chenliping1950@126.com
}


tion (QSE), or quantitative autonomic examination (QAE), one of which is NC or QAE) are sufficient to diagnose DPN. The Toronto Diabetic Neuropathy Expert Group recommends that the diagnosis of DPN should be mainly based on symptoms, signs, and nerve conduction studies (NCS), and the measurement of small fibre neuropathy (SFN) [6].

NCS and the measurement of SFN are considered sensitive, objective, and reproducible approaches for assessing DPN. However, due to limited conditions (funding, laboratory, and invasiveness), the wide use of NCS and measurement of SFN has remained unrealistic. Therefore, the routine diagnosis of DPN has been based on clinical symptoms and signs, which are simpler approaches that can be used in an outpatient setting.

Clinically, the symptoms and signs of DPN can be quantified using validated scoring systems, such as NSS, NDS, [4, 5, 7] Toronto clinical scoring system (TCSS) [8, 9], and Michigan Neuropathy Screening Instrument (MNSI) [10].The evaluation of tendon reflexes is included in these clinical scores. In clinical practice, tendon reflexes have been used to evaluate DPN. Decreased or absent tendon reflexes are among the most common signs that suggest the possibility of DPN.

Hence, the aim of the present study was to evaluate the diagnostic efficacy of different tendon reflexes in detecting DPN.

\section{Material and methods}

From March 1, 2015, to December 31, 2017, patients with type-2 diabetes mellitus, who had regular follow-ups in the Diabetes Clinic of the Second Hospital of Hebei Medical University, were randomly selected for the present study. Two neurologists carefully evaluated each patient to exclude patients with other causes of DPN, such as alcohol abuse, exposure to neurotoxic drugs, endocrine, and inflammation. The present study was conducted in accordance with the Declaration of Helsinki and approved by the Ethics Committee of The Second Hospital of Hebei Medical University. Written informed consent was obtained from all participants.

All patients were carefully interviewed and underwent neurological examinations, which included the assessment of conventional reflexes (biceps reflex, triceps reflex, radial periosteal reflex, patella reflex, and Achilles tendon reflex), mental status, cranial nerve function, sensory tests for a light touch, pin-prick, vibration and joint position sense, muscle strength, balance, gait, stretch reflexes, and coordination. The most common changes in tendon reflexes in diabetic patients were normal tendon reflexes, impaired (decreased or absent) Achilles tendon reflex only, impaired lower extremity tendon reflexes (Achilles tendon reflex and pa- tella reflex), and impairment of all tendon reflexes (upper and lower extremity tendon reflexes).

According to the characteristics of the lengthdependent DPN, the changes in tendon reflex can be divided into three strata. In stratum 1, the Achilles tendon reflex decreased or was absent, including the impaired Achilles tendon reflex only, impaired lower extremity tendon reflexes, and impairment of all tendon reflexes. In stratum 2, lower extremity tendon reflexes decreased or were absent, which included impaired lower extremity tendon reflexes and the impairment of all tendon reflexes. In stratum 3, all tendon reflexes decreased or were absent.

All patients were clinically assessed using the TCSS and MNSI. The TCSS was originally proposed by Perking $[8,9]$. These patients were scored according to the score for symptoms (foot pain, numbness, tingling, weakness, imbalance, and upper limb symptoms), the score for tendon reflexes (bilateral patella and Achilles tendon reflexes), and the score for sensory signs (pin-prick, temperature, light touch, vibration, and position sense). Grading was stratified as follows: a score of 5 indicates no neuropathy, a score within 6-8 indicates mild neuropathy, a score within 9-11 indicates moderate neuropathy, and a score of 12 indicates severe neuropathy. The MNSI questionnaire was followed by a simple eight-point clinical examination, which involved the inspection of the foot, assessment of Achilles tendon reflexes, and semiquantitative determination of vibration perception. An MNSI score of $>2$ indicated the presence of neuropathy [10].

The nerve conduction velocity of all patients was measured via electromyography by skilled neurophysiologists. Standardised techniques for NCS with temperature control and fixed distances were applied. The measurement of latencies, distances, and amplitudes was performed in the standard fashion using onset latencies and baseline-to-peak amplitudes. For sensory curves, initial positive peak (if present) to negative peak measurements were used. The conduction velocities were automatically calculated by the Medtronic Keypoint EMG equipment. The F-wave program installed in the Medtronic Keypoint EMG equipment was used. F-waves were elicited by 20 supramaximal stimuli to measure the minimum and maximum latencies and calculate the mean latency for nerves. Then, the motor (median, ulnar, tibial, and peroneal) and sensory (median, ulnar, and sural) nerves were measured, and the data for compound muscle action potential (CMAP)/sensory nerve action potential (SNAP) and motor conduction velocity $(\mathrm{MCV})$ /sensory conduction velocity (SCV) were obtained. Comprising stimulation of the median and ulnar nerves at the wrist (S1) and elbow (S2), 
and tibial and peroneal nerves at the ankle (S1) and knee (S2), the CMAP was recorded with the active electrode placed on the belly of the abductor pollicis brevis, abductor digiti minimi, abductor hallucis, and extensor digitorum brevis, and the reference electrode was placed $3 \mathrm{~cm}$ more distally. Another surface electrode pasted on the forearm or leg served as a ground. The following formula was used: $M C V=$ distance $(\mathrm{S} 2-\mathrm{S} 1)$ /latency $(\mathrm{S} 2-\mathrm{S} 1)$. Stimulation of the median and ulnar nerves at the wrist (S), and sural nerves at the posterolateral calf (S). SNAP was recorded with the active electrode placed on the metacarpophalangeal joint of fingers I and V, Posterior malleolus (R). The following formula was used: SCV = distance $(S-R) /$ latency R. According to NCS, the patients were divided into a normal NCS group and an abnormal NCS group. The tendon reflexes were divided into three groups: normal tendon reflexes, impaired Achilles tendon reflex only, and impaired lower extremity tendon reflexes (patella reflex and Achilles tendon reflex), to further study the relationship between tendon reflex and NCS.

\section{Sensitivity and specificity}

The sensitivity, specificity, and diagnostic accuracy of the tendon reflexes were calculated, with NCS as the gold standard. The true-positive, truenegative, false-positive, and false-negative findings were evaluated, and standard formulas were used to estimate the sensitivity, specificity, and diagnostic accuracy.

\section{Statistical analysis}

The results were analysed using the Statistical Package for Social Sciences (SPSS, Shanghai, China) version 17.0. Pearson's $\chi^{2}$ test or independent samples $t$-test was used to examine the differences between different variables, as appropriate. The Shapiro-Wilk test was used to check the normal distribution of the examining variable before the $t$-test. In cases of non-normal distributions of the variable, the Mann-Whitney test was used. Skewed values were log-transformed before the analysis. A $p$-value of $<0.05$ was considered statistically significant.

\section{Results}

A total of 240 patients with diabetes were enrolled in this study. Among these patients, 123 (51.3\%) were female, while 117 (48.7\%) were male. The mean age of these patients was $56.5 \pm 12.4$ years, and the disease duration of 110 patients was more than 10 years (45.8\%). The mean value of glycated haemoglobin $\left(\mathrm{HbA}_{1 \mathrm{c}}\right)$ was $(8.26 \pm 1.67) \%$, while the value of the body mass index (BMI) was $(26.06 \pm 3.87) \mathrm{kg} / \mathrm{m}^{2}$. Furthermore, abnormal NCS was observed in 92 (38.3\%) patients, while normal NCS was observed in 148 (61.7\%) patients. The impaired Achilles tendon reflexes were observed in $40(16.7 \%)$ patients, impaired lower extremity tendon reflexes were observed in 76 (31.6\%) patients, impairment of all tendon reflexes was observed in 37 (15.4\%) patients, and normal tendon reflexes were observed in 87 (36.3\%) patients. The mean values for TCSS and MNSI were $4.25 \pm 3.55$ and $0.90 \pm 1.12$, respectively. The clinical and demographic characteristics of the abnormal and normal NCS groups are presented in Table I. The TCSS scores revealed mild to severe neuropathy in $68.5 \%$ and $20.9 \%$ of patients in the abnormal NCS group and the normal NCS group, respectively ( $p<0.001$ ). Similarly, $47.8 \%$ of patients in the $a b-$ normal NCS group and $17.6 \%$ of patients in the normal NCS group had neuropathy, as determined by MNSI $(p<0.001)$. The metabolic parameters in the two groups are shown in Table II. A higher $\mathrm{HbA}_{1 c}$ level was observed in patients with abnormal NCS $(p=0.008)$.

The sensitivity and specificity of the reflexes and screening tests are shown in Table III. Taking NCS as the gold standard, stratum 1 yielded a sensitivity and specificity of $93.5 \%$ and $54.7 \%$, respectively. Stratum 3 had higher specificity (96.6\%), but lower sensitivity (34.8\%), when compared to

Table I. Baseline characteristics of patients in the two groups

\begin{tabular}{|c|c|c|c|}
\hline Parameter & $\begin{array}{l}\text { Abnormal } \\
\text { NCS group } \\
(n=92)\end{array}$ & $\begin{array}{l}\text { Normal } \\
\text { NCS group } \\
(n=148)\end{array}$ & $P$-value \\
\hline Age [years] & $58.6 \pm 10.94$ & $55.21 \pm 13.10$ & $0.035^{b}$ \\
\hline \multicolumn{4}{|l|}{ Gender: } \\
\hline Male & 42 & 75 & $0.449^{a}$ \\
\hline Female & 50 & 73 & \\
\hline \multicolumn{4}{|l|}{$\begin{array}{l}\text { Duration } \\
\text { of diabetes } \\
\text { [years]: }\end{array}$} \\
\hline $1-10$ & 0 & 90 & $0.001^{a}$ \\
\hline$>10$ & 52 & 58 & \\
\hline \multicolumn{4}{|l|}{ TCSS: } \\
\hline$<6$ & 29 & 117 & $<0.0001^{a}$ \\
\hline$\geq 6$ & 63 & 31 & \\
\hline \multicolumn{4}{|l|}{ MNSI: } \\
\hline$\leq 2$ & 8 & 122 & $<0.0001^{\mathrm{a}}$ \\
\hline$>2$ & 44 & 26 & \\
\hline
\end{tabular}

${ }^{a} \chi^{2}$ test, ${ }^{b}$ independent samples $t$-test. NCS - nerve conduction studies, TCSS - Toronto clinical scoring system, MNSI - Michigan Neuropathy Screening Instrument. 
Table II. Baseline characteristics of patients in the two groups

\begin{tabular}{|lccc|}
\hline Parameter & $\begin{array}{c}\text { Abnormal } \\
\text { NCS group } \\
(n=92)\end{array}$ & $\begin{array}{c}\text { Normal } \\
\text { NCS group } \\
(n=148)\end{array}$ & $P$-value \\
\hline BMI $\left[\mathrm{kg} / \mathrm{m}^{2}\right]$ & $26.26 \pm 4.71$ & $25.93 \pm 3.24$ & 0.611 \\
\hline $\mathrm{HbA}{ }_{1 \mathrm{c}}(\%)$ & $8.86 \pm 1.98$ & $8.28 \pm 1.87$ & 0.008 \\
\hline LDL-c $[\mathrm{mmol} / \mathrm{l}]$ & $2.73 \pm 1.13$ & $2.64 \pm 0.83$ & 0.704 \\
\hline $\mathrm{TG}[\mathrm{mmol} / \mathrm{l}]$ & $2.29 \pm 1.3$ & $2.38 \pm 1.81$ & 0.843 \\
\hline $\mathrm{HDL}-\mathrm{c}[\mathrm{mmol} / \mathrm{l}]$ & $1.15 \pm 0.43$ & $1.07 \pm 0.41$ & 0.570 \\
\hline TC $[\mathrm{mmol} / \mathrm{l}]$ & $5.43 \pm 2.03$ & $4.61 \pm 1.29$ & 0.069 \\
\hline
\end{tabular}

Data are means $\pm S D$. BMI - body mass index, $H b A_{1 c}$ - glycated haemoglobin, HDL-c - high-density lipoprotein cholesterol, LDL-C - low-density lipoprotein cholesterol, TG - triglyceride, TC - total cholesterol. stratum 1. However, stratum 2 had the highest specificity $(83.7 \%$ and $75.7 \%$ for sensitivity and specificity, respectively).

The relationship between nerve conduction velocity and tendon reflexes was investigated (Table IV). The minimum latency of the F-wave in the group of abnormal lower extremities reflexes was longer when compared to that in the group of normal tendon reflex ( $61.3 \pm 5.6$ vs. $45.0 \pm 4.3$, $p=0.001)$ and the group of abnormal Achilles (61.3 \pm 5.6 vs. $57.0 \pm 6.3, p=0.017)$. In addition, the latency F-wave in the group of abnormal Achilles was longer, when separately compared to that in the group of normal tendon reflex and the group of abnormal Achilles ( $45.0 \pm 4.3$ vs. $57.0 \pm 6.3$, $p=0.025)$. The SNAP and SCV of the sural nerve in

Table III. Sensitivity and specificity of tendon reflex and other screening tests with NCS as gold standard

\begin{tabular}{|c|c|c|c|c|c|c|c|}
\hline Variable & TP & FP & $\mathrm{FN}$ & $\mathrm{TN}$ & Sensitivity (\%) & Specificity (\%) & $\begin{array}{c}\text { Diagnostic } \\
\text { accuracy (\%) }\end{array}$ \\
\hline Stratum 1 & 86 & 67 & 6 & 81 & 93.5 & 54.7 & 69.6 \\
\hline Stratum 2 & 77 & 36 & 15 & 112 & 83.7 & 75.7 & 78.8 \\
\hline Stratum 3 & 32 & 5 & 60 & 143 & 34.8 & 96.6 & 72.9 \\
\hline TCSS & 63 & 31 & 29 & 117 & 68.5 & 79.1 & 75.0 \\
\hline MNSI & 44 & 26 & 48 & 122 & 47.8 & 82.4 & 69.2 \\
\hline
\end{tabular}

$T P$ - true positive, FP - false positive, FN - false negative, $T N$ - true negative.

Table IV. Electrophysiological characteristics of diabetic patients with different tendon reflexes

\begin{tabular}{|c|c|c|c|c|c|c|c|}
\hline \multicolumn{2}{|c|}{ Characteristics } & \multicolumn{2}{|c|}{ Abnormal } & \multirow{2}{*}{$\begin{array}{c}\text { Normal } \\
\text { Tendon reflex }\end{array}$} & \multicolumn{3}{|c|}{$P$-value } \\
\hline & & $\begin{array}{l}\text { Lower extremities } \\
\text { tendon reflexes ( } 1)\end{array}$ & $\begin{array}{c}\text { Achilles reflex } \\
\text { (2) }\end{array}$ & & 1 vs. 2 & 1 vs. 3 & 2 vs. 3 \\
\hline \multirow[t]{2}{*}{ Median } & SNAP & $17.3 \pm 10.8$ & $18.1 \pm 12.4$ & $20.5 \pm 14.7$ & 0.334 & 0.265 & 0.221 \\
\hline & SCV & $54.8 \pm 3.2$ & $55.1 \pm 2.8$ & $52.7 \pm 4.8$ & 0.136 & 0.531 & 0.537 \\
\hline \multirow[t]{2}{*}{ Ulnar } & SNAP & $8.1 \pm 4.8$ & $8.5 \pm 5.1$ & $9.3 \pm 6.8$ & 0.246 & 0.428 & 0.445 \\
\hline & SCV & $57.1 \pm 5.8$ & $59.1 \pm 3.7$ & $56.5 \pm 4.5$ & 0.226 & 0.276 & 0.111 \\
\hline \multirow[t]{2}{*}{ Sural } & SNAP & $2.0 \pm 0.8$ & $2.5 \pm 1.2$ & $4.5 \pm 2.3$ & 0.031 & 0.001 & 0.048 \\
\hline & SCV & $38.5 \pm 4.7$ & $45.1 \pm 5.3$ & $56.7 \pm 5.9$ & 0.015 & 0.001 & 0.018 \\
\hline \multirow[t]{2}{*}{ Median } & CMAP & $5.5 \pm 1.8$ & $6.1 \pm 2.1$ & $6.4 \pm 2.3$ & 0.087 & 0.095 & 0.108 \\
\hline & MCV & $57.1 \pm 2.4$ & $60 \pm 5.8$ & $64 \pm 4.4$ & 0.161 & 0.061 & 0.278 \\
\hline \multirow[t]{2}{*}{ Ulnar } & CMAP & $8.7 \pm 1.6$ & $9.6 \pm 1.2$ & $8.4 \pm 2.1$ & 0.160 & 0.128 & 0.504 \\
\hline & MCV & $58 \pm 4.3$ & $64 \pm 6.1$ & $65 \pm 5.7$ & 0.066 & 0.045 & 0.094 \\
\hline \multirow[t]{2}{*}{ Tibial } & CMAP & $13.3 \pm 5.4$ & $14.5 \pm 4.9$ & $20.6 \pm 6.9$ & 0.012 & 0.001 & 0.057 \\
\hline & MCV & $39.0 \pm 3.2$ & $45.1 \pm 2.8$ & $51 \pm 4.2$ & 0.016 & 0.001 & 0.032 \\
\hline \multirow[t]{2}{*}{ Peroneal } & CMAP & $3.8 \pm 1.9$ & $4.6 \pm 2.7$ & $5.2 \pm 4.9$ & 0.103 & 0.194 & 0.156 \\
\hline & MCV & $50.0 \pm 3.6$ & $48.2 \pm 3.7$ & $49.2 \pm 5.9$ & 0.943 & 0.838 & 0.387 \\
\hline \multicolumn{2}{|c|}{$\begin{array}{l}\text { F wave (Tibial) } \\
\text { Minimum latencies }\end{array}$} & $61.3 \pm 5.6$ & $57.0 \pm 6.3$ & $45.0 \pm 4.3$ & 0.017 & 0.001 & 0.025 \\
\hline
\end{tabular}


the group of abnormal lower extremities reflexes was less than those in the group of normal tendon reflexes $(2.0 \pm 0.8$ vs. $4.5 \pm 2.3$ and $38.5 \pm 4.7$ vs. 56.7 $\pm 5.9, p=0.001$ and $p=0.001$, respectively) and the group of abnormal Achilles ( $2.0 \pm 0.8$ vs. $2.5 \pm 1.2$ and $38.5 \pm 4.7$ vs. $45.1 \pm 5.3, p=0.031$ and $p=0.015$, respectively). The SCV of the sural nerve in the group of abnormal Achilles was less than that in the group of normal tendon reflexes $(2.5 \pm 1.2$ vs. $4.5 \pm 2.3$ and $45.1 \pm 5.3$ vs. $56.7 \pm 5.9, p=0.048$ and $p=0.018$, respectively). The CMAP and MCV of the tibial nerve in the group of abnormal lower extremities reflexes were less than that in the group of normal tendon reflexes $(13.3 \pm 5.4$ vs. $20.6 \pm 6.9$ and $39.0 \pm 3.2$ vs. $51 \pm 4.2, p=0.001$ and $p=0.001$, respectively) and the group of abnormal Achilles $(13.3 \pm 5.4$ vs. $14.5 \pm 4.9$ and $39.0 \pm 3.2$ vs. $45.1 \pm 2.8$, $p=0.012$ and $p=0.016$, respectively).

\section{Discussion}

In the present study, we found that the changes in tendon reflexes were closely correlated to the incidence of DPN. Tendon reflexes could indicate DPN when there is a lack of NCS and measurement of the small fibre nerve. Furthermore, the Achilles tendon reflex and patella reflex display the most acceptable sensitivity, specificity, and predictive values. Thus, assessment of tendon reflexes could be proposed as a test for screening diabetic polyneuropathy.

The main purpose of screening for DPN is to treat it effectively in the early stages. At present, confirming DPN requires NCS or the measurement of SFN. However, the wide use of NCS and measurement of SFN remains limited because testing is time-consuming and costly, and there is limited availability of specialised laboratories. The efficacy of TCSS and MNSI scores has been validated for DPN in other studies [8-10]. However, these scores are too complex, time-consuming, and not suitable for screening DPN in diabetes or primary care clinics. As a result, physicians cannot routinely use these methods.

Impaired tendon reflexes are common in patients with DPN. In the present study, impaired tendon reflexes were found in $63.7 \%$ of patients with diabetes. Tendon reflex is a common and objective testing method, which can indirectly reflect the degree of injury of the peripheral nerve in neurological clinical practice. Decreased or absent deep tendon reflexes often indicate mild and asymptomatic DPN. An absent Achilles tendon reflex is considered the most sensitive index for screening DPN [11]. However, an absent Achilles tendon reflex has often been observed in healthy subjects, particularly in subjects aged $>70$ years, because S1 nerve injury is induced by the atherosclerotic obstruction of the vasa vasorum or nutri- tional deficiency [12]. Because many factors influence the Achilles tendon reflex, the false-positive rate of the Achilles tendon reflex in the diagnosis of DPN is higher.

In clinical practice, it has been found that the absence of Achilles tendon reflexes is often accompanied by the absence of patella reflexes in subjects with diabetes. Age does not affect the patella reflex, and the intervertebral disc seldom squeezes the L2-L4 nerve root. Thus, the patella reflex may be more powerful than the Achilles tendon reflex when screening for DPN. However, it remains to be determined whether the diagnostic efficacy of an absent or decreased patella, or Achilles tendon reflexes, are higher than merely an absent or decreased Achilles tendon reflex.

In this study, we found that the sensitivity of stratum 1 was higher than that of stratum 2 (93.5\% and $83.7 \%$, respectively), while the specificity of stratum 2 was much higher than that of stratum 1 (75.7\% and 54.7\%, respectively). In addition, the diagnostic accuracy of stratum 2 was significantly higher than that of stratum 1 (78.8\% and $69.6 \%$, respectively). Thus, in the present study, the Achilles tendon reflex and patella reflex display the most acceptable sensitivity, specificity, and predictive values.

Based on the above findings, we conducted a further study to determine whether impaired lower extremity tendon reflexes were consistent with NCS. These tendon reflexes were divided into three groups: normal tendon reflexes, impaired Achilles tendon reflex only, and impaired lower extremity tendon reflexes. Our data showed that the changes in tendon reflexes were consistent with those in the neuroelectrophysiology group. The more weakened or absent the tendon reflex was, the more serious the impairment of the nerve conduction became. The F-wave is one of the most sensitive tools for diagnosing peripheral neuropathy [13], especially for DPN [14]. In the present study, when the ankle reflex decreased or was absent, the latency of the F-wave was prolonged. When the knee reflex decreased or was absent, the latency of the F-wave was further prolonged. Sural sensory nerves and tibial motor nerves are most susceptible to DPN. For DPN, nerve conduction velocity is more sensitive than amplitude. In the present study, when the ankle reflex was only impaired, the velocity of the sural nerve slowed down, but not the amplitude. Studies have shown that the sural nerve pathology in DPN was paranodal abnormalities of segmental demyelination and remyelination without axonal degeneration [15].

According to the above test results, incidences of DPN are closely correlated to changes in tendon reflexes. Tendon reflexes could indicate DPN 
when there is a lack of NCS and measurement of the small fibre nerve. If an Achilles tendon reflex is present in a diabetic patient, the possibility of DPN can be excluded. If all tendon reflexes are absent or diminished, DPN can probably be diagnosed. To our knowledge, our study is the first study to recommend the absence or decrease of Achilles tendon and patella reflexes as a screening tool for DPN, because this method can quickly and effectively distinguish DPN.

The present study has several limitations. First, it was a single-centre retrospective study, so selective bias cannot be avoided. Second, the number of patients was not adequate to draw an accurate conclusion. Furthermore, the sensory changes and the correlation with deep tendon reflexes were not evaluated in our patients. The correlation between tendon reflexes and the severity of peripheral nerve injury was also not evaluated.

In conclusion, our study demonstrated that the assessment of tendon reflexes could be proposed as a test for screening for diabetic polyneuropathy.

\section{Conflict of interest}

The authors declare no conflict of interest.

\section{References}

1. Petrovic M, Maganaris CN, Deschamps K, et al. Altered Achilles tendon function during walking in people with diabetic neuropathy: implications for metabolic energy saving. J Appl Physiol 2018; 124: 1333-40.

2. Ma CC, Duan CC, Huang RC, Tang HQ. Association of circulating cystatin $\mathrm{C}$ levels with type 2 diabetes mellitus: a systematic review and meta-analysis. Arch Med Sci 2019; 16: 648-56.

3. Hussain N, Adrian TE. Diabetic neuropathy: update on pathophysiological mechanism and the possible involvement of glutamate pathways. Curr Diabetes Rev 2017; 13: 488-97.

4. Dyck P, Kratz K, Lehman K, et al. The Rochester Diabetic Neuropathy Study: design, criteria for types of neuropathy, selection bias, and reproducibility of neuropathic tests. Neurology 1991; 41: 799-807.

5. Dyck PJ, Karnes JL, O'Brien PC, Litchy WJ, Low PA, Melton LJ III. The Rochester diabetic neuropathy study: reassessement of tests and criteria for diagnosis and staged severity. Neurology 1992; 42: 1164-70.

6. Tesfaye S, Boulton AJ, Dyck PJ, et al.; Toronto Diabetic Neuropathy Expert Group. Diabetic neuropathies: update on definitions, diagnostic criteria, estimation of severity, and treatments. Diabetes Care 2010; 33: 2285-93.

7. Tentolouris A, Eleftheriadou I, Grigoropoulou P, et al. The association between pulse wave velocity and peripheral neuropathy in patients with type 2 diabetes mellitus. J Diabetes Complications 2017; 31: 1624-9.

8. Perkins BA, Olaleye D, Zinman B, Bril V. Simple screening tests for peripheral neuropathy in the diabetes clinic Diabetes Care 2001; 24: 250-6.

9. Bril V, Perkins BA. Validation of the Toronto Clinical Scoring System for diabetic polyneuropathy. Diabetes Care 2002; 25: 2048-52
10. Seferovic JP, Pfeffer MA, Claggett B, Desai AS, Zeeuw DD, Haffner SM. Three-question set from Michigan Neuropathy Screening Instrument adds independent prognostic information on cardiovascular outcomes: analysis of ALTITUDE trial. Diabetologia 2018; 61: 581-8.

11. Shehab DK, Al-jarallah KF, Abraham M, Mojiminiyi OA, Al-mohamedy $\mathrm{H}$, Abdella NA. Back to basics: Achilles tendon reflex in the evaluation of peripheral neuropathy in type 2 diabetes mellitus. Q J Med 2012; 105: 315-20.

12. Bowditch MG, Sanderson P, Livesey JP. The significance of absent patella reflex. J Bone Joint Surg B 1996; 78 : 276-9.

13. Jerath NU, Aul E, Reddy CG, Azadeh H, Swenson A, Kimura J. Prolongation of F-wave minimal latency: a sensitive predictor of polyneuropathy. Int I Neurosci 2016; 126 520-5.

14. Pan H, Jian F, Lin J, et al. F-wave latencies in patients with diabetes mellitus. Muscle Nerve 2014; 49: 804-8.

15. Malik RA, Tesfaye S, Newrick PG, et al. Sural nerve pathology in diabetic patients with minimal but progressive neuropathy. Diabetologia 2005; 48: 578-85. 